\title{
Use of SEVIRI images and derived products in a WMO Sand and Dust Storm Warning System
}

\author{
M A Martínez, J Ruiz and E Cuevas \\ Agencia Estatal de Meteorología (AEMET), Spain \\ E-mail: mig@inm.es
}

\begin{abstract}
The Visible/IR images of SEVIRI (Spinning Enhanced Visible and Infrared Imager), on board Meteosat Second Generation (MSG) satellites, are used to monitor dust events. Satellite-based detection of dust is a difficult problem due in part to the observing-system limitations. The main difficulty is that the dust can be confused with water/ice clouds. SEVIRI is not as optimal for the viewing of dust as SEAWIFS or MODIS, due to the fact that both of them count with additional short-wavelength channels. However, the SEVIRI 15-minute loop images can detect small dust plumes as well as subtle changes from one image to the next. A description of how the AEMET, former INM, is developing the environment to support MSG satellite imagery to the WMO/GEO Sand and Dust Storm Warning System (SDS WS) for Europe, Africa and Middle East Regional Centre will be briefly presented, together with some on-going operational developments to best monitor dust events.
\end{abstract}

\section{Introduction}

This paper is embedded in the activities of the SDSWS for Europe, Africa and Middle East Regional Centre. The region covered by this SDSWS Centre is shown in figure 1, being mostly inside the Meteosat satellites coverage.

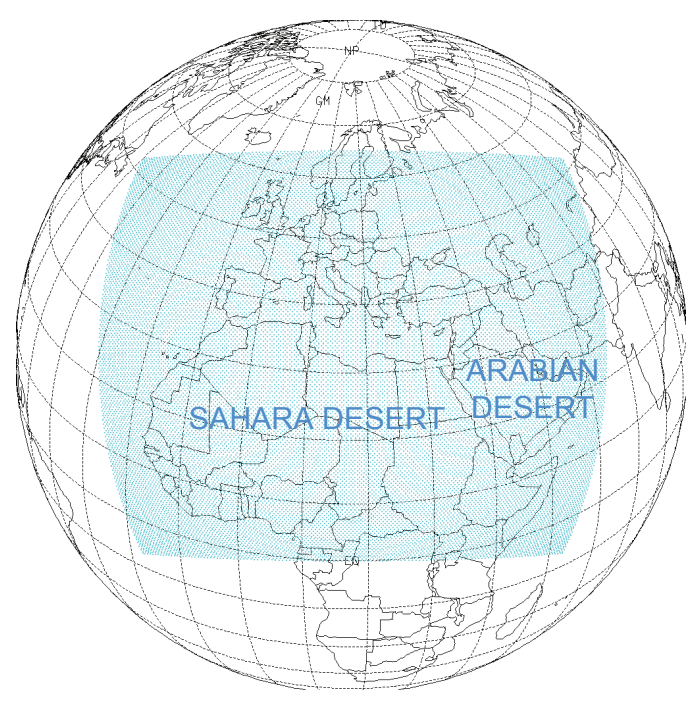

Figure 1. Region covered by the SDSWS 
METEOSAT-9, which belongs to the Meteosat Second Generation series, is the current nominal satellite. The main instrument on board MSG satellites is the SEVIRI radiometer. The eight thermal IR $(3.9,6.2,7.3,8.7,9.7,10.8,12.0,13.4 \mu \mathrm{m})$ and the three solar channels $(0.6,0.8,1.6 \mu \mathrm{m})$ have a sampling distance of three kilometers at nadir and scan the full disk of the Earth. The high-resolution visible channel provides images with one kilometer sampling distance at nadir. Data rate limitations confine the high-resolution visible images to half the Earth disk in an east-west direction; however, the exact coverage of the Earth is programmable. Moreover, METEOSAT-8 started providing Rapid Scan Service (RSS) in May 2008 taking images every 5 minutes and covering approximately a latitude range of $15^{\circ}$ to $69^{\circ} \mathrm{N}$; this service allow monitoring dust events with unprecedented temporal resolution.

EUMETSAT is developing two MPEF day-2 aerosols related products, within its Meteorological Product Extraction Facility (MPEF). On the other hand, EUMETSAT Satellite Application Facilities for Nowcasting and Very Short Range Forecasting (NWC SAF) software package provides a dust product. This product is included in the Cloud Mask product as a flag that indicates the presence of dust. As the rest of NWC SAF products, it is obtained in near real time, at full resolution $(3 \mathrm{~km} \times 3 \mathrm{~km}$ at nadir) every cycle of 15 minutes for the region selected by the user. More information about this can be obtained in reference 3 or in NWC SAF web page (http://www.nwcsaf.org).

This paper is focused on the use of SEVIRI images in the monitoring of dust storm events. First, it will be shown the implementation of the RGB images recommended by EUMETSAT for monitoring desert dust with MSG. Later, some early developments made at the AEMET to enhance the analysis of dust information contained in the SEVIRI VIS channels will be presented.

\section{Continuous monitoring of desert dust using EUMETSAT RGB images}

EUMETSAT recommends the use of some RGB images for dust monitoring in the MSG interpretation guide which is available on its web page (http://oiswww.eumetsat.org/WEBOPS/msg_interpretation/ index.html). The Dust RGB is an RGB composition based upon infrared channel data from SEVIRI (table 1) where dusty pixels appear in pink colors. It is designed to monitor the evolution of dust storms over deserts during both day and night. Dust RGB images are available free of charge every hour through the EUMETSAT web page: http://www.eumetsat.int/Home/Main/Image_Gallery/ Real_Time_Imagery/index.htm.

Table 1. Dust RGB composition

\begin{tabular}{cccccc}
\hline \multicolumn{7}{c}{ Dust (night \& day) } \\
\hline $\begin{array}{c}\text { RGB colour } \\
\text { plane }\end{array}$ & $\begin{array}{c}\text { Channel } \\
\text { (difference) }\end{array}$ & MIN & MAX & GAMMA & $\begin{array}{c}\text { Prominent } \\
\text { features }\end{array}$ \\
\hline $\mathbf{R}$ & $12.0-10.8$ & $-4 \mathrm{~K}$ & $+2 \mathrm{~K}$ & 1.0 & $\begin{array}{c}\text { Dust(over land) } \\
\text { Thin Cirrus } \\
\text { Contrails }\end{array}$ \\
\hline $\mathbf{G}$ & $10.8-8.7$ & $0 \mathrm{~K}$ & $+15 \mathrm{~K}$ & 2.5 & \\
\hline $\mathbf{B}$ & 10.8 & $261 \mathrm{~K}$ & $289 \mathrm{~K}$ & 1.0 & \\
\hline
\end{tabular}

The Natural RGB is an RGB composition based upon visible channels data from SEVIRI (table 2). It is recommended for the tracking of dust across the oceans during the day.

Table 2. Dust RGB composition

\begin{tabular}{cccccc}
\hline $\begin{array}{c}\text { RGB colour } \\
\text { plane }\end{array}$ & Channel & MIN & MAX & GAMMA & $\begin{array}{c}\text { Prominent } \\
\text { features }\end{array}$ \\
\hline $\mathbf{R}$ & 1.6 & $0 \%$ & $100 \%$ & 1.0 & Ice/water clouds, fog, snow \\
\hline $\mathbf{G}$ & 0.8 & $0 \%$ & $100 \%$ & 1.0 & $\begin{array}{c}\text { Dust(over ocean),smoke } \\
\text { (Green) vegetation }\end{array}$ \\
\hline $\mathbf{B}$ & 0.6 & $0 \%$ & $100 \%$ & 1.0 & C
\end{tabular}


The strongest sand storm monitored with MSG satellite took place in March 2004 and it has been selected as a clear example. The intensity and the wide extension affected this dust event can be appreciated in the Dust RGB image shown in figure 2 (3rd March at 12Z). The dust event affected the whole Sahara desert and crossed the Atlantic Ocean in the following days. Loops of dust RGB images for this event are available on EUMETSAT and on WMO WS web page (http://salam.upc.es/wmo). On the other hand, the dust over ocean can be seen in the Natural RGB image shown in figure 3 (5th March at 9Z).

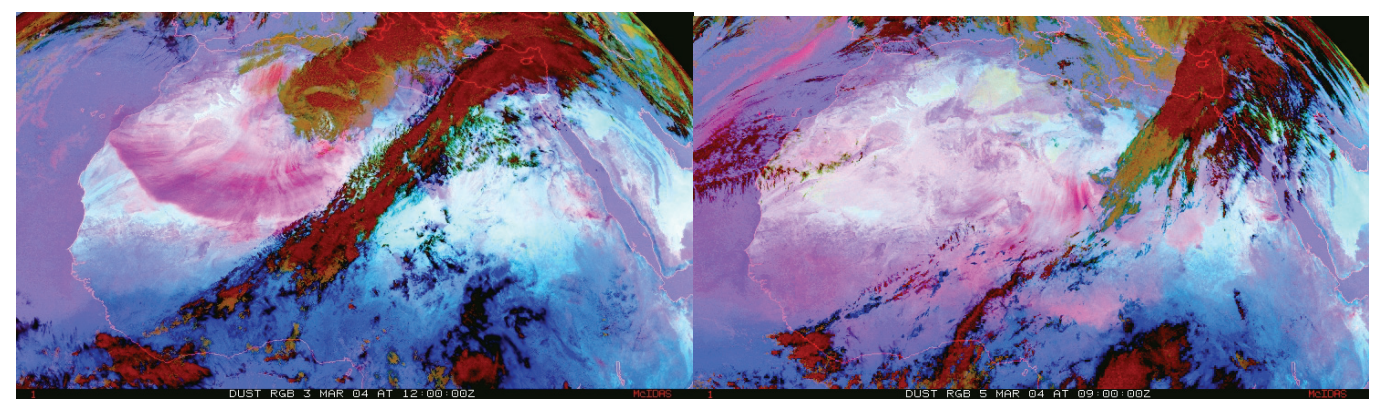

Figure 2. Dust RGB images during March 2004 dust event. 3rd March $200412 Z$ (left) and $5^{\text {th }}$ March 2004 9Z (right)

\section{AEMET development (The current Approach)}

\subsection{Normalized natural RGB}

Due to the fact that the incoming radiance is different depending on the position of the earth throughout the year, and also depending on the moment the satellite makes the observation. It will be convenient to normalize in a certain manner the radiance values obtained by the radiometer in order to compare situations that can last several days and that can appear at any time during the year.

The Bidirectional Reflectance Factor (BRF) (equation 1) allows to correct the visible radiances (RAD) taking into account the solar zenith angle (SZA), the Sun Earth distance (SE), the channel bandwidth (BW) and the Sun Irradiance (SI).

$$
B R F=\frac{\pi \cdot R A D \cdot B W \cdot S E^{2}}{S I \cdot \cos (S Z A)}
$$

The BRF variable factors can be grouped in one term, called normalized Radiance in this paper (equation 2):

$$
R A D n=\frac{R A D \cdot S E^{2}}{\cos (S Z A)}
$$

The normalized radiance for the $0.6,0.8$ and $1.6 \mu \mathrm{m}$ SEVIRI channels is routinely calculated using a command programmed in the McIDAS (Man computer Interactive Data Access System) environment (http://www.ssec.wisc.edu/mcidas). This command has been developed at the AEMET. The coefficients for the calculation of SZA and SE have been obtained from [1]. The Natural RGB command is applied to the normalized radiances and the obtained RGB images are called normalized Natural RGB images.

In figure 3, the Natural RGB image (left) is compared with the normalized Natural RGB image (right) for the same slot. The normalized Natural RGB shows a better dust monitoring, to simple sight. 
It also allows discriminating dust during more hours (sunrise to sunset). Moreover, it is possible to compare normalized Natural RGB images throughout the year.

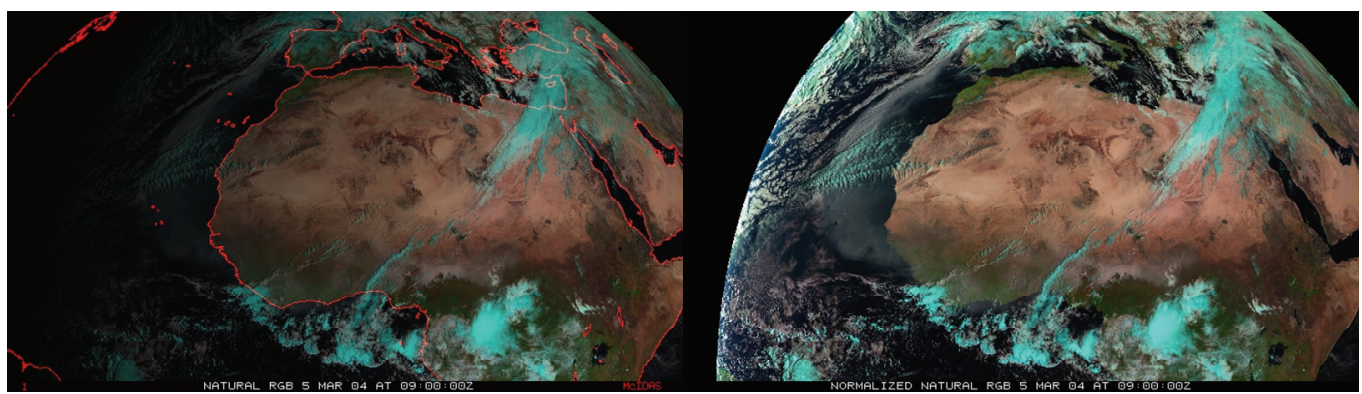

Figure 3. Natural RGB images during March 2004 dust event. Not normalized on the left and normalized on the right (5 March 2004 9Z)

\subsection{Dust monitoring over land pixels using SEVIRI visible channels}

Over land pixels the visible channels are not so efficient in the dust monitoring. The $19^{\text {th }}-25^{\text {th }}$ February 2007 sand storm event has been selected to investigate the information contained in the visible channels over land pixels. The image of NWC SAF dust flag for the 21 st February 2007 at $12 \mathrm{Z}$ is shown in figure 4, where pixels in green represent zones where the algorithm has detected the presence of dust.

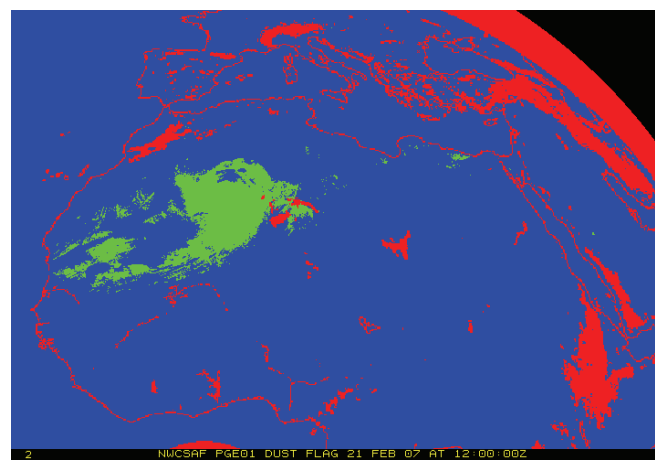

Figure 4. Dust flag of SAFNWC Cloud Mask (21 February 2007 at 12Z). Dusty pixels appear green

In figure 5, the Dust RGB image (on the left), with dusty pixels in pink color, is shown together with the normalized radiances of each visible channel (on the right) for the same date and time. On the visible channels over the region of interest (red square), it can be seen how the presence of the dust is mixed with the ground information.
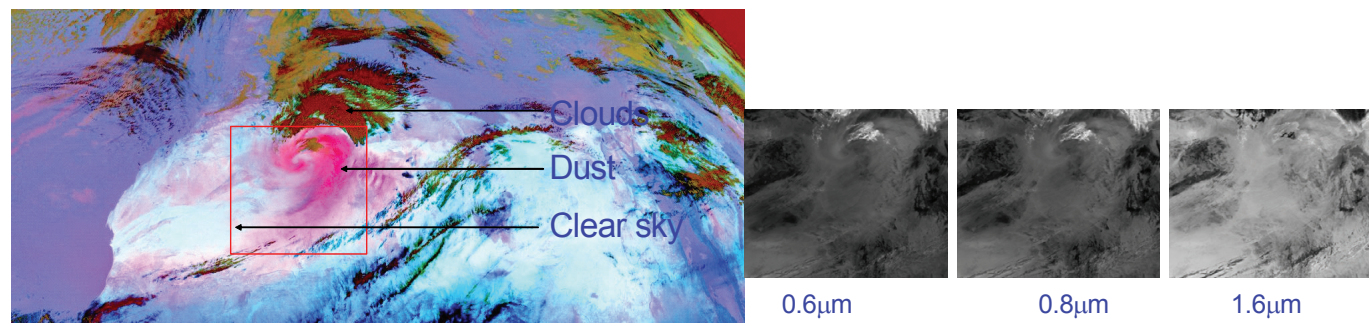

Figure 5. Dust RGB and 0.6, 0.8 and 1.6 Normalized Radiance inside the red square region (21 February 2007 12Z) 
The objective is to detect the presence of dust in the visible SEVIRI images. In order to reach it, it is necessary to detach the information of the background introduced by the textures of the desert, and to enhance the information coming from the dust. This task has been performed building monthly cloudless radiance masks at $12 \mathrm{Z}$ for each channel $(0.6,0.8,1.6 \mu \mathrm{m})$. The used criteria to construct the monthly mask for each channel are different for land and sea pixels. Over every land pixel, the array containing the radiances for the month is sorted (in ascending order). Later on, the day with the highest NDVI is searched among the third part of the data with lower values (excluding the minimum value). In the case of sea pixels, all the data for the month is managed together and the value with the maximum frequency of the histogram for the month is selected. All the sea pixels are set to this fixed value.

The normalized Natural RGB image for the dust event of the $21^{\text {st }}$ February is shown in figure 6 (right); the dust appears as a shadow in the image. When the Natural RGB is applied to the masks, the ground information is retained by the monthly mask. As an example the February 2007 RGB mask image is shown in figure 6 (left).

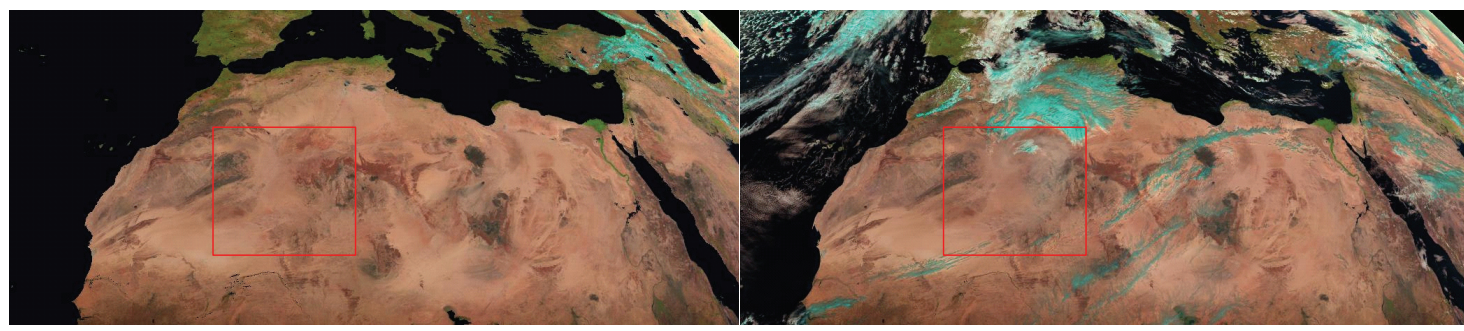

Figure 6. Normalized Natural RGBs: monthly mask (left) and 21 February 2007at 12Z (right)

\subsection{Prototype of dust classifier}

Detection of dust over bright land such as deserts is more difficult than over ocean, and it requires IR information to separate the dust signal from the other components of the scene. In the Dust RGB image of figure 7 (left) it can be seen some variations in the dust intensity (different pink colour intensity). Four classes have been established (figure 7, right) following the physical basis of the Dust RGB: high intensity (white), medium intensity (yellow), low intensity (red) and clear-sky (blue). Table 3 contains the empirical thresholds used to separate the classes.
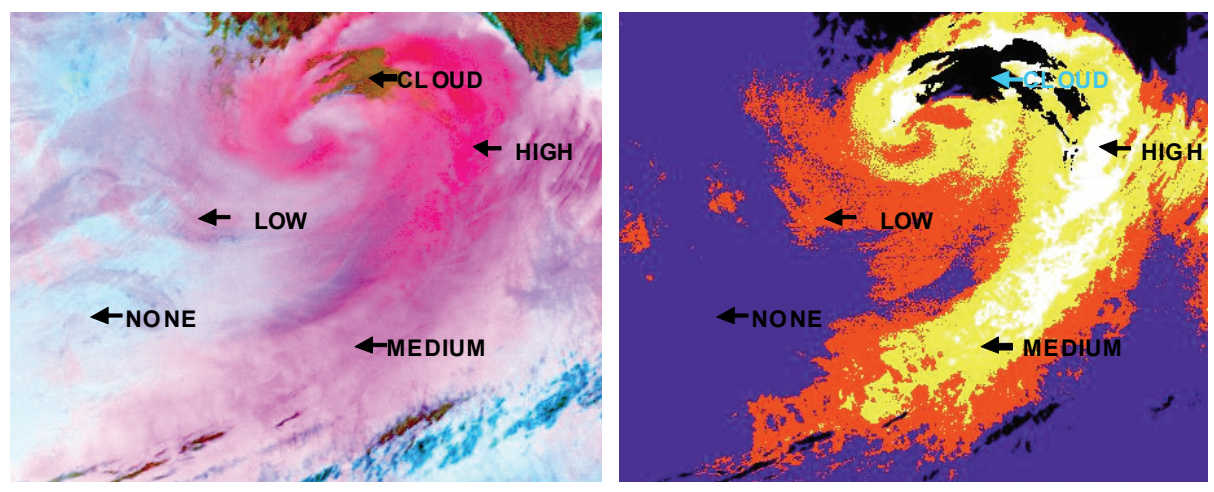

Figure 7. Dust RGB 21st February 2007 12Z (left) and classification of dust intensity (right)

The main issue is to study the dust signal on the visible channels. The BRF scatter-plot of each visible channel versus the corresponding BRF monthly mask for each class have been analyzed; in figure 8, the VIS0.6 scatter-plots are shown as an example. 
Table 3. Criteria used to obtain the dust intensity classification.

\begin{tabular}{cccccc}
\hline & HIGH & MEDIUM & LOW & CLOUD & NONE \\
\hline IR12.0-IR10.8 & $>3 \mathrm{~K}$ & $>1.9 \mathrm{~K}$ & $>1 \mathrm{~K}$ & -- & Other case \\
\hline IR10.8-IR8.7 & $<2 \mathrm{~K}$ & $<4 \mathrm{~K}$ & $<7 \mathrm{~K}$ & -- & Other case \\
\hline IR10.8 & -- & -- & -- & $<275 \mathrm{~K}$ & Other case \\
\hline
\end{tabular}
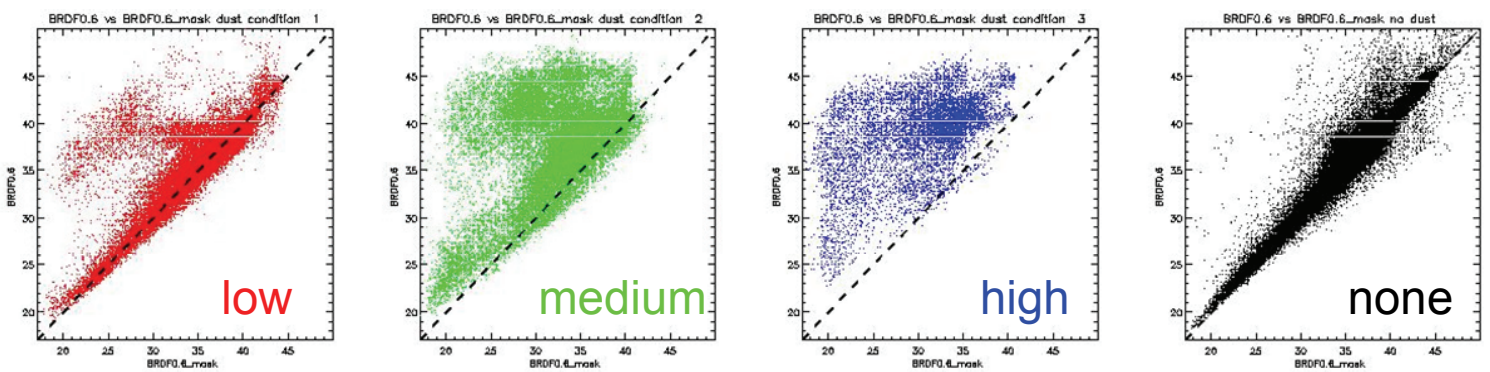

Figure 8. Scatter-plot of BRF VIS0.6 versus the BRF VIS0.6 monthly mask for each class of dust intensity.

Differences between the image and its monthly mask can been detected in all channels for medium and high intensity classes. As the differences between the image and the mask are small, the use of the logarithm of the difference is more adequate to enhance the presence of dust. In figure 9, it is shown an RGB composition with the logarithm of the differences between each BRF and its mask (following the same Natural RGB channel order). It can be seen how the textures of the desert have disappeared and how the regions affected by the presence of dust are clearly enhanced.

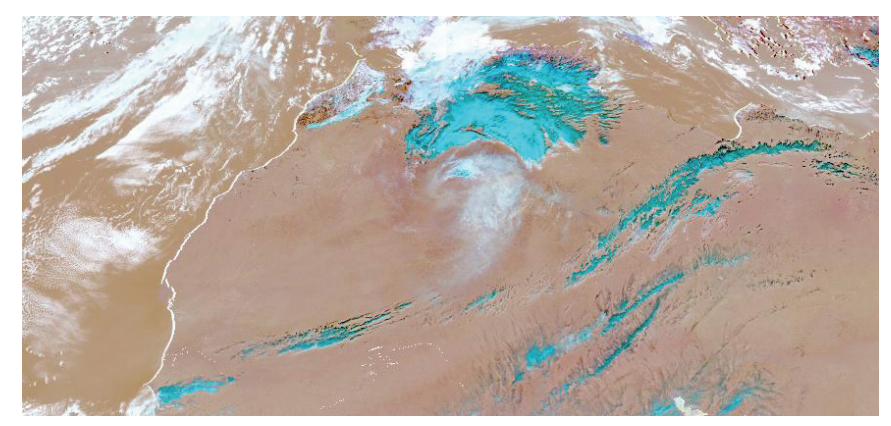

Figure 9. RGB composition with the logarithm of the differences between each BRF and its mask (21Ffebruary 2007 $12 \mathrm{Z})$.

\section{Conclusions}

The SEVIRI spectral range, its spatial resolution ( $3 \mathrm{~km}$ in the IR bands at nadir), and its 15 minutes cycle allow to better monitor desert dust events. The RGB images constitute an easy and fast approach for dust monitoring. The used of visible normalized radiances improve the dust monitoring when the Natural RGB is applied. The thermal infrared thresholds used in the preliminary classification have been obtained empirically and could be improved in future works. An operational environment system for dust monitoring based on web pages is being developed at the AEMET. However, further work is needed to improve the quality of the dust estimation using visible channels over land pixels.

Satellite data can complement dust models and in situ measurements to allow a better monitoring of dust events. In particular, the comparison with DREAM numerical model outputs (http://www.bsc.es/projects/earthscience/DREAM) is included in the SDSWS project.

\section{References}

[1] Liou, K.N., 2002 An introduction to Atmospheric Radiation. Second Edition. International Geophysics Series. Volume 84. Academic Press. Pages 44-50. 